\title{
PEPTÍDEOS DE COLÁGENO COMBINADO AO TIPO II NA DOR ARTICULAR DO IDOSO
}

\section{ARTIGO ORIGINAL}

LIMA, Rafaela Batista de ${ }^{1}$

AMARAL, Camilla Lais ${ }^{2}$

MINATTI, Jaqueline ${ }^{3}$

LIMA, Rafaela Batista de. AMARAL, Camilla Lais. MINATTI, Jaqueline. Peptídeos de colágeno combinado ao tipo II na dor articular do idoso. Revista Científica Multidisciplinar Núcleo do Conhecimento. Ano 05, Ed. 08, Vol. 07, pp. 115-127. Agosto de

2020.

ISSN:

2448-0959,

Link

de

acesso: https://www.nucleodoconhecimento.com.br/nutricao/peptideos-de-colageno

\section{RESUMO}

Sabe-se que o colágeno é uma proteína de grande importância para os ossos, tendões, cartilagem, músculos, pele, cabelo e unhas. Existem vários tipos de colágeno, sendo que, o tipo II é o principal componente da matriz da cartilagem articular e pode ajudar a prolongar o estilo de vida independente de um idoso, melhorando a sua qualidade de vida. A pesquisa objetivou avaliar o efeito de um mix de colágenos composto por peptídeos, colágeno tipo II e nutrientes envolvidos no metabolismo ósseo articular de idosos ativos. Participaram da pesquisa voluntários com idade mínima de 60 anos e fisicamente ativos. Trata-se de um ensaio clínico nãorandomizado, realizado com o grupo de ginástica dos idosos de um Centro de

\footnotetext{
${ }^{1}$ Bacharel em Nutrição no Centro Universitário Estácio de Santa Catarina, Brasil.

${ }^{2}$ Mestre em nutrição. Docente do curso de Nutrição do Centro Universitário Estácio de Santa Catarina, Brasil, Orientadora.
}

${ }^{3}$ Mestre em nutrição. Coorientadora. 
Atividades em Florianópolis - SC. A suplementação oral com o mix de colágeno composto por peptídeos bioativos de colágeno hidrolisado, colágeno tipo II intacto, silício e nutrientes envolvidos no metabolismo ósseo articular (Duo flex®) foi administrado por 90 dias, sendo sempre entregue o próximo ciclo de 30 dias do suplemento antes de findar o ciclo atual, afim de que os participantes não interrompessem o tratamento. Estes também eram semanalmente acompanhados via contato telefônico para evitar a desistência ou interrupção do tratamento. Foram aplicados questionários para avaliar os sintomas relacionados a sarcopenia, as funcionalidades das articulações, a característica da saúde da pele, unhas e cabelos e medidas antropométricas. A amostra foi constituída por 50 idosos, a maioria eram do sexo feminino (72\%). O consumo do produto reduziu a dor, diminuiu a rigidez e melhorou a mobilidade das articulações conforme o resultado do questionário WOMAC. O Duo flex® melhorou significativamente a saúde das articulações e, portanto, pode ser uma solução eficaz para retardar as manifestações articulares. Os resultados sugerem que o mix de colágeno pode ser usado como uma abordagem terapêutica para idosos fisicamente ativos.

Palavras-Chave: colágeno, idoso, osteoartrite, composição corporal, envelhecimento.

\section{INTRODUÇÃO}

Conforme a Organização das Nações Unidas (ONU, 2017), sistemas de cuidados de saúde em todo o mundo estão trabalhando para promover o envelhecimento saudável, prevenir e tratar doenças crônicas não transmissíveis. A população global com 60 anos ou mais totalizou 962 milhões em 2017, mais do dobro que em 1980, quando havia 382 milhões de pessoas idosas em todo o mundo. Espera-se que o número de idosos continue a crescer consideravelmente até 2050 , quando se prevê atingir quase 2,1 bilhões.

O indivíduo idoso pode apresentar manifestações clínicas com diversas alterações que fazem parte do envelhecimento natural mesmo tendo uma vida fisicamente ativa e alimentação saudável. Com isso ocorrem perda gradual de massa muscular, degenerações nas cartilagens articulares, envelhecimento da pele, queda de cabelo, 
unhas fracas, o que pode, assim, diminuir a satisfação com a vida e aumentar a taxa de mortalidade (CAI, 2016).

A cartilagem articular é um tecido bem organizado com durabilidade notável, entretanto, o dano pode resultar em dor articular debilitante e comprometimento funcional na pessoa idosa, piorando a mobilidade (MORAN et al., 2014; QUEIROZ; LEMOS; RAMOS, 2010). Segundo Bakilan et al. (2016), alterações degenerativas são observadas tanto na cartilagem articular quanto no osso subcondral. O desequilíbrio entre a síntese e a degradação leva à sua destruição. Portanto, numerosos agentes farmacêuticos e nutricionais foram desenvolvidos com o objetivo de retardar a progressão das mudanças estruturais na Osteoartrite $(\mathrm{OA})$, como o colágeno.

O colágeno é uma proteína de função estrutural para as células e existem diferentes tipos de colágeno, dependendo dos tecidos dos quais se origina. O tipo I é o mais comum, geralmente encontra-se em locais que resistem a grandes tensões como nos tendões, na derme, nos ossos e até mesmo na córnea. Já o tipo II é encontrado em estruturas resistentes a grandes pressões, como cartilagem elástica e hialina, discos intervertebrais e nos olhos. O colágeno tipo III é identificado na artéria aorta, nos pulmões, nos músculos do intestino, fígado, no útero que constitui as fibras reticulares e o tipo VI, não se associa a fibrilas, tem a função de sustentação e filtração, presente nos rins, na lâmina basal e na cápsula do cristalino (GERMANO et al., 2016).

Os peptídeos de colágeno (PC) foram propostos como nutracêuticos para melhorar a saúde das articulações em pacientes com osteoartrite. Em estudo prospectivo, randomizado, duplo-cego, controlado por placebo em mulheres idosas com osteoartrite leve a moderada do joelho, foi demonstrado que a ingestão oral de peptídeos de colágeno durante seis meses reduziu significativamente a dor nas articulações e melhorou a mobilidade avaliada por dois sistemas de pontuação bem estabelecidos (escore WOMAC e Lysholm). Este estudo confirmou que os peptídeos de colágeno são nutracêuticos altamente eficientes para melhorar a saúde das articulações, o que pode ajudar a manter um estilo de vida ativo ao longo do envelhecimento (JIANG et al., 2014). 
Visto que o organismo do indivíduo idoso necessita de cuidados para que sejam minimizadas as agressões aos tecidos articular, ósseo e muscular para que tenham maior autonomia e melhor qualidade de vida, o objetivo do presente estudo foi avaliar o efeito de um mix de colágeno, composto por peptídeos de colágeno, colágeno tipo II, silício orgânico e nutrientes envolvidos no metabolismo ósseo articular dos idosos.

\section{DESENVOLVIMENTO}

\subsection{METODOLOGIA}

Trata-se de um ensaio clínico não-randomizado, com participantes que frequentavam as aulas de ginástica, turma de volei, treinamento multifuncional e pilates em um Centro de Atividades na cidade de Florianópolis - SC. Foram recrutados indivíduos com idade igual ou superior a 60 anos. Os voluntários participaram do estudo somente após a assinatura do Termo de Consentimento Livre e Esclarecido (TCLE) conforme a Resolução nª 466/12 do Conselho Nacional de Saúde, o qual foi aprovado pelo Comitê de Ética em Pesquisas (CEP) do Centro Universitário Estácio de Santa Catarina, atendendo as exigências da resolução $n^{\circ}$ 466/12, a qual descreve os direitos que são estabelecidos para os projetos de pesquisa envolvendo seres humanos. Aprovado conforme o processo (CAAE: 96708718.0.0000.5357).

O estudo incluiu 50 sujeitos afetados com dores articulares e/ou diagnóstico autoreferido da doença óssea e/ou articular. Os sujeitos começaram o tratamento de 90 dias com o mix de colágeno composto por peptídeos bioativos de colágeno hidrolisado, colágeno tipo II intacto, silício e nutrientes envolvidos no metabolismo ósseo articular (Duo flex®) com a finalidade de avaliar a influência dessa suplementação no estado geral dos participantes, especialmente nas manifestações articulares.

A pesquisa contou com os seguintes critérios de exclusão: participantes que estavam utilizando a condroitina e/ou glucosamina, anti-inflamatórios não esteroidais (AINEs) e corticoesteroides, mas aqueles que estivam utilizando esses mesmos fármacos a mais de 30 dias e ainda sentiam dores, foram incluídos. Participantes que não 
cumprissem o protocolo ou que modificassem hábitos alimentares e/ou físicos durante a intervenção poderiam ser excluídos do estudo. Também seriam excluídos aqueles que fizessem cirurgias, injeções de medicamentos intra-articulares, ou que tivessem modificado medicação via oral no mês prévio ao início do estudo. Participantes com sinovite e derrame no joelho, doenças sistêmicas concomitantes graves, distúrbio neurológico periférico ou central, insuficiência cardíaca, renal, hepática ou doença hematológica grave não foram aceitos na pesquisa. Além daqueles que inserissem medicações como condroitina e/ou glucosamina, (AINEs) e corticoesteroides no decorrer do estudo, também seriam excluídos.

Os dados foram coletados no tempo 0, após 30, 60 e 90 dias de intervenção.

\subsection{DESENHO METODOLÓGICO}

Para traçar o perfil da amostra foi utilizado o questionário adaptado com variáveis como idade, gênero, nível de atividade física e medidas antropométricas (MOTA, 2017).

Com relação aos dados antropométricos, o peso foi aferido por meio de uma balança digital de vidro transparente da marca Powner® com capacidade de $150 \mathrm{~kg}$. Para aferição da altura, foi utilizado o estadiômetro compacto de $200 \mathrm{~cm}$, fixado na parede, da marca Slim $\mathrm{Fit} \circledast$. O estado nutricional dos idosos foi classificado segundo o Índice de Massa Corporal (IMC) e avaliado conforme os pontos de corte da Organização Pan-americana de Saúde (OPAS 2002), sendo os idosos classificados como baixo peso (IMC $\leq 23 \mathrm{~kg} / \mathrm{m}^{2}$ ), peso normal (IMC $\geq 23 \leq 28 \mathrm{~kg} / \mathrm{m}^{2}$ ), excesso de peso (IMC $\left.\geq 28 \leq 30 \mathrm{~kg} / \mathrm{m}^{2}\right)$ e Obesidade $\left(\mathrm{IMC} \geq 30 \mathrm{~kg} / \mathrm{m}^{2}\right)$.

Para investigar o efeito da suplementação nos sintomas relacionados a sarcopenia foi aplicado o escore SARC-F + CP (circunferência da panturrilha), que contêm 5 componentes que significam as letras do nome deste questionário em inglês, Força, Assistência com caminhada, Levante-se de uma cadeira, Suba escadas e Quedas, e as pontuações variam de 0 a 10 para a $\mathrm{CP}$ e de 0 a 2 pontos para cada componente 
que compõe o questionário. A pontuação igual ou maior que 4 foi preditivo de sarcopenia (MALMSTROM et al., 2016).

Para a medida da circunferência da panturrilha, utilizou-se uma fita métrica da marca TBW® de plástico flexível, inelástica, auto retrátil e graduada de 0 a $150 \mathrm{~cm}$, aferido na parte mais protuberante, enquanto o paciente estava sentado, com a perna dobrada em ângulo de 90 graus. A circunferência da panturrilha é um bom parâmetro de avaliação da massa muscular no idoso e deve ser considerado adequado a circunferência igual ou superior a $31 \mathrm{~cm}$ para homens e mulheres (BRASIL, 2017).

O escore HAQ (Health Assessment Questionnaire - Questionário de Avaliação de Saúde) avalia oito áreas de atividades funcionais habituais, com 20 perguntas sobre a vida cotidiana do paciente. Os pacientes responderam às perguntas cada qual com quatro possibilidades de respostas, sendo elas: nenhuma dificuldade (escore 0), com alguma dificuldade (escore 1), com muita dificuldade (escore 2) e incapaz de realizar (escore 3). Calculou-se a média aritmética do maior escore de cada uma das oito áreas avaliadas e o resultado final foi o escore do HAQ (FERNANDES, 2003).

O escore WOMAC (Westerm Ontário and McMaster Universities) é dividido por seções que questionam a intensidade da dor na artrose do joelho, intensidade de rigidez articular no joelho e habilidade para locomover-se e para cuidar-se nas atividades físicas diárias, no qual as repostas podem ser "nenhuma", "leve", "moderada", "forte", "muito forte". Para analisar os dados, estes níveis foram transformados respectivamente em $0,25,50,75$ e 100. Portanto, utilizadas para analisar a eficácia da suplementação na recuperação das articulações (FERNANDES, 2003).

O escore Lequesne também foi utilizado para analisar as articulações e segundo Marx et al. (2006), investiga se a pessoa sente dor, desconforto ou não sente dor durante as atividades diárias. É uma forma de avaliar a melhora funcional de um paciente com osteoartrite, onde o sujeito relata suas dificuldades. As pontuações variam de zero (sem acometimento) a dois (incapaz) e a soma da pontuação varia de 1 a 4 (pouco acometimento), 5 a 7 (moderada), 8 a 10 (grave), 11 a 13 (muito grave), igual ou maior que 14 (extremamente grave). 
O questionário do dia usual descreveu as refeições diárias de cada sujeito, para controle dos hábitos alimentares durante o estudo. Foram considerados adequados dietas com valores entre 90 a $110 \%$ da recomendação, insuficiente quando $<90 \%$ e excessivo quando $>110 \%$. Além disso, foi aplicado um questionário adaptado utilizado para avaliar as características da saúde da pele, unhas e cabelos como forma indireta de avaliar os possíveis benefícios dos peptídeos de colágeno e associações do suplemento sobre a saúde das estruturas corporais. Após 30, 60 e 90 dias de ingestão do tratamento, com perguntas que avaliaram se houve diferença quanto à firmeza, elasticidade, hidratação e aspecto geral da pele, quanto à aparência, crescimento $e$ força das unhas (ADDOR, 2015).

\subsection{INTERVENÇÃO}

Os participantes foram instruídos a ingerir diariamente $12 \mathrm{~g}$ do Duo flex da marca

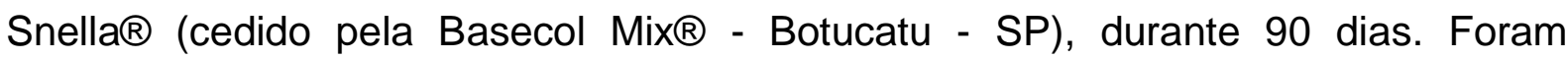
orientados a dissolver o produto em um copo de água, suco ou vitamina ou ainda, amassado com frutas num dos intervalos entre as principais refeições (lanche). Os indivíduos foram instruídos sobre os benefícios da ingestão do suplemento e da necessidade de manter sem alterações os hábitos alimentares e físicos, bem como o uso de medicamentos durante o estudo.

Os dados foram tabulados no programa Microsoft Office $\mathrm{Excel}^{\circledR} \mathrm{e}$ analisados com o software GraphPad InStat com Teste T de Student, sendo considerado significante $p<0,05$. Os resultados obtidos foram apresentados através de tabelas e gráficos.

\subsection{RESULTADOS E DISCUSSÃO}

Dos idosos analisados, a maioria eram do sexo feminino (72\%). A média de idade foi de $67 \pm 5,2$ anos, sem diferença significativa entre os sexos, sendo média de $67 \pm 4,9$ anos nas mulheres e média de $67 \pm 6,3$ anos nos homens. A altura média dos voluntários foi de $1,61 \pm 0,096 \mathrm{~m}$ e o peso médio foi de $71 \pm 12,7 \mathrm{~kg}$. Quanto ao tipo de atividade física dos participantes, 16\% praticavam ginástica, 36\% vôlei, $24 \%$ 
treinamento multifuncional e $24 \%$ pilates. Todos os participantes se mantiveram ativos fisicamente durante o estudo e não alteraram suas medicações e/ou alimentação.

A avaliação do estado nutricional (EN) dos participantes mostrou que 16\% estavam baixo peso, a maior parcela (58\%) apresentavam peso normal, $12 \%$ apresentavam excesso de peso e 14\% estavam em obesidade. Os participantes mantiveram o mesmo EN até o fim do estudo.

Em relação ao EN, o elevado peso (26\% dos participantes), somados aos processos naturais e desgastes do envelhecimento, podem interferir na manutenção da saúde com o aumento das dores articulares, influenciando negativamente na qualidade de vida e na morbimortalidade (CHUMLEA, 1989).

Outra queixa comum observada na amostra estudada foi quanto à saúde da pele, unhas e cabelos. Sabe-se que a redução de colágeno, situação comum na senectude, não só piora a saúde das estruturas osseoarticulares, mas também se mostra nas questões estéticas. Quanto a avaliação das características da pele, unhas e cabelo, houve uma melhora parcial das unhas em $32 \%$ dos analisados; melhora parcial de unhas e cabelo (26\%); melhora parcial de pele e unha (4\%); melhora total do cabelo $(10 \%)$; melhora total de cabelo, pele e unha (6\%); e relataram indiferença $(22 \%)$ dos estudados. Conforme o aumento da idade há mudanças que levam à diminuição do colágeno na população em geral, que ainda não são totalmente compreendidas. O envelhecimento pode variar de pessoa para pessoa, estilo de vida, doenças atuais e as já estabelecidas, influências genéticas e ambientais (YAAR et al., 2002), sendo que a reposição de peptídeos de colágeno parece trazer resultados benéficos independentemente de fatores externos, no presente estudo, visto em $78 \%$ dos analisados.

Para avaliação da sarcopenia através o protocolo SARC-F + CP, foi evidenciado que $16 \%$ dos idosos iniciaram no t0 com sarcopenia, já no t30 passaram a ser $14 \%$ e ao final do estudo passou para 12\%. Apesar da suplementação não ter o objetivo principal voltado à melhora da massa magra, ainda assim ao final do estudo $4 \%$ dos participantes evidenciaram esses resultados benéficos. Não houve novos casos de 
sarcopenia durante a pesquisa. O estudo de Zdzieblik (2015), pôde comprovar que os indivíduos suplementados com colágeno apresentaram um aumento maior na massa magra e força muscular, o que corrobora os resultados em questão.

Além da análise da massa magra, foi feita a análise do estado funcional avaliado por meio do $\mathrm{HAQ}$, onde mais da metade $(60 \%)$ apresentaram-se na classificação de deficiência leve, ou seja, que são capazes de realizar as atividades de vida diária, entretanto, houve uma prevalência de $47 \%$ que relataram dores ou desconforto na articulação do ombro, e que conforme a figura 1, observa-se o desempenho ao longo do t30 ( $p=0,0003)$, t60 ( $p<0,0001)$ e t90 ( $p<0,0001)$ quando comparados ao t0. Ultimamente, peptídeos de colágeno têm sido vividamente discutidos como agente modificador de sintomas para osteoartrite. Sediada na sua aplicação em alimentos funcionais como ingredientes bioativos, eles são pensados para agir em uma fase antes da doença, ajudando a prevenir ou atrasar a manifestação de osteoartrite (VIJVEN et al. 2012), quanto em situações já estabelecidas, trazendo melhoras.

Figura 1 - Comparação da dor/desconforto mensurada por Questionário de HAQ (Média \pm DP) durante os tempos do estudo com a suplementação do mix de colágeno.

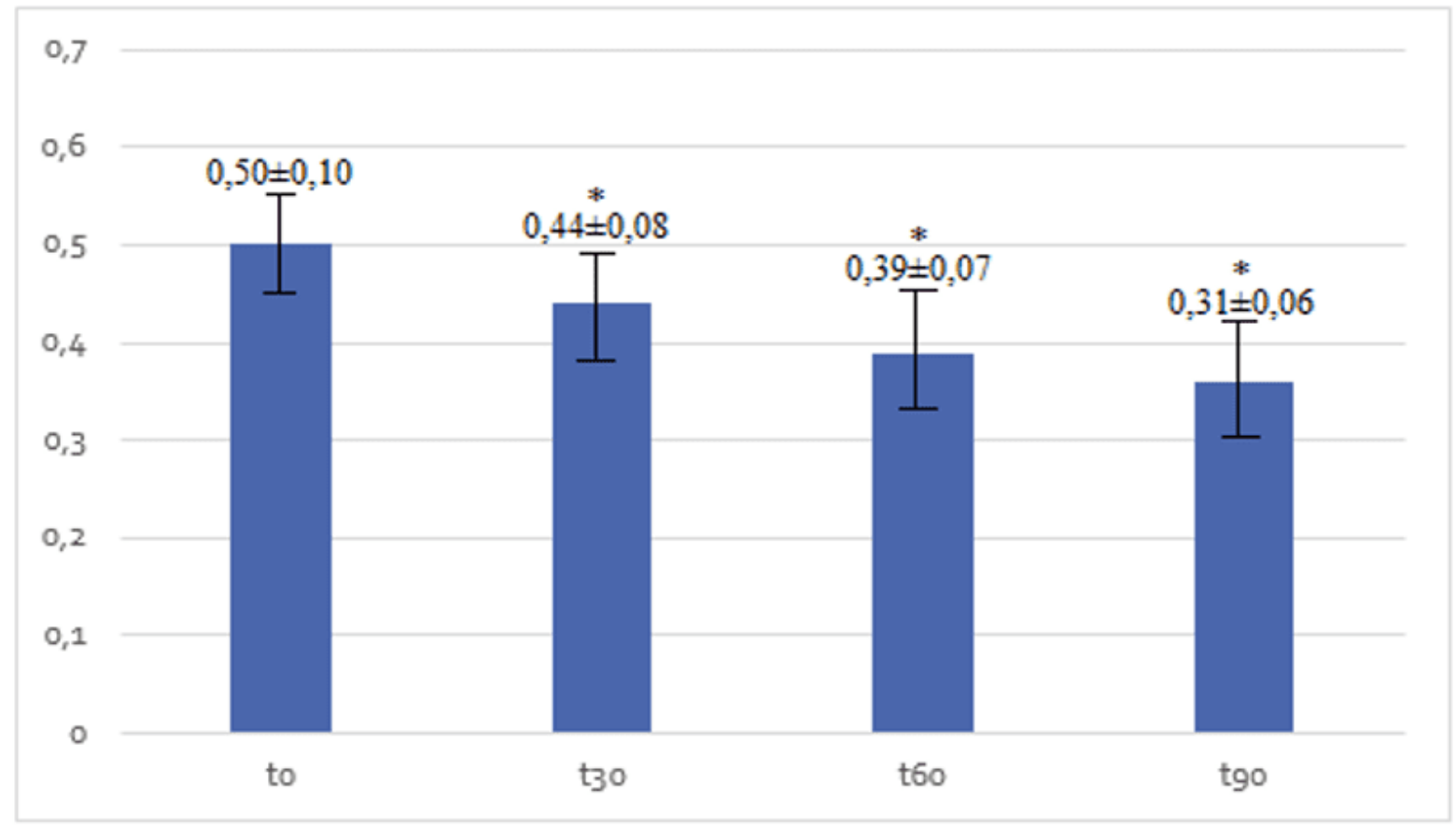

Fonte: dados primários (2018). Legenda: ${ }^{*} \mathrm{p}<0,05$ quando comparado ao t0. 
$\mathrm{Na}$ avaliação do protocolo WOMAC observou-se que 58\% dos voluntários eram portadores de osteoartrite no joelho ou sentiam algum desconforto relacionado aos sintomas. Para os participantes que responderam este protocolo, foi obtido já no primeiro mês de tratamento uma melhora, conforme demonstrado na figura 2, com a melhora da capacidade funcional no $\mathrm{t} 30(\mathrm{p}=0,0001)$, t60 ( $\mathrm{p}<0,0001)$ e t90 $(\mathrm{p}<0,0001)$. Estes resultados corroboram com os achados no estudo Crowley et al. (2009) com a demonstração da eficácia do tratamento com o colágeno tipo II e redução do escore WOMAC em $33 \%$ relacionadas as dificuldades nas atividades diárias dos indivíduos.

Figura 2 - Comparação da capacidade funcional mensurada por WOMAC (Média \pm DP) durante os tempos do estudo com a suplementação do mix de colágeno.

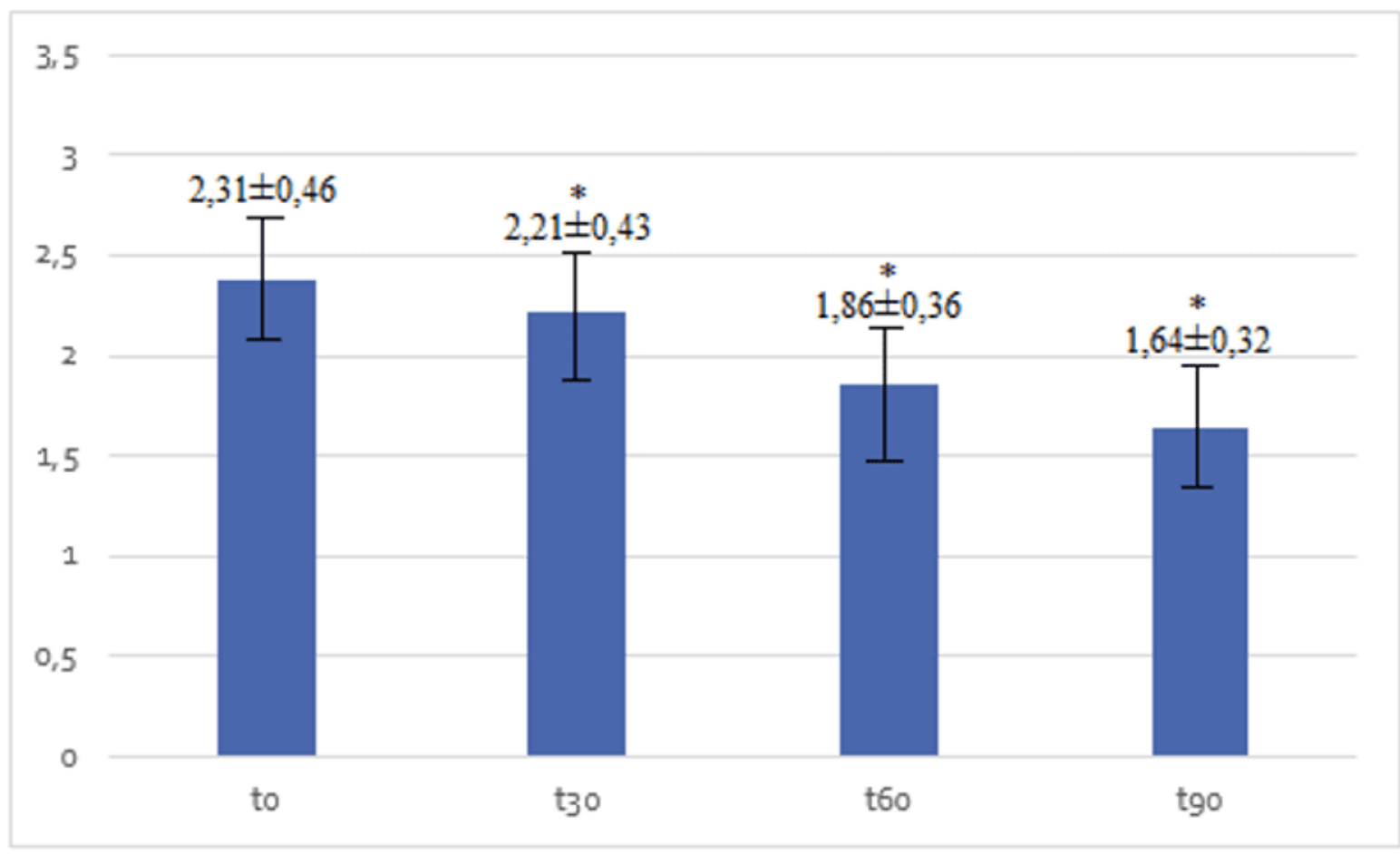

Fonte: dados primários (2018). Legenda: ${ }^{*} \mathrm{p}<0,05$ quando comparado ao t0.

Segundo Lugo et al. (2015), também evidenciaram que indivíduos que consumiram colágeno tipo II apresentaram melhores resultados clínicos em comparação aos suplementados com placebo neste estudo. A análise das subescalas WOMAC mostrou que houve reduções nas três subescalas WOMAC contribuindo para a 
melhoria na pontuação geral do WOMAC observada em indivíduos suplementados com colágeno tipo II

Sabe-se que com o avanço do envelhecimento, há perda de osso trabecular. No entanto, de acordo com o estudo de Oftadeh et al. (2015), a microarquitetura trabecular foi melhorada após o tratamento com PC. Este resultado sugere claramente que os PC são eficazes para melhorar a capacidade da rede trabecular.

Quanto a classificação de dor do escore Lequesne a maioria do sexo masculino $(71 \%)$ idade média $65 \pm 5,2$ anos apresentou alguma dificuldade na atividade diária em relação ao joelho e quadril, sendo que, 30\% apresentavam obesidade segundo o IMC o que pode influenciar neste resultado agravando as lesões. Conforme figuras 3 , houve redução da dor/desonforto no quadril no t30 ( $p<0,0001)$, t60 ( $p<0,0001)$ e t90 $(p<0,0001)$ e no joelho no t30 ( $p<0,0001), t 60(p<0,0001)$ e t90 $(p<0,0001)$. A utilização do colágeno pode ser usado na prevenção e/ou no tratamento da osteoartrite e da osteoporose. Estudo clínico, mostrou eficiência na diminuição de dores articulares e melhora da mobilização de pacientes quando suplementados (ZIEGLER e SGARBIERI, 2009).

Figura 3a - Comparação da dor/desconforto no quadril mensurada por Lequesne (Média \pm DP) durante os tempos do estudo com a suplementação do mix de colágeno.

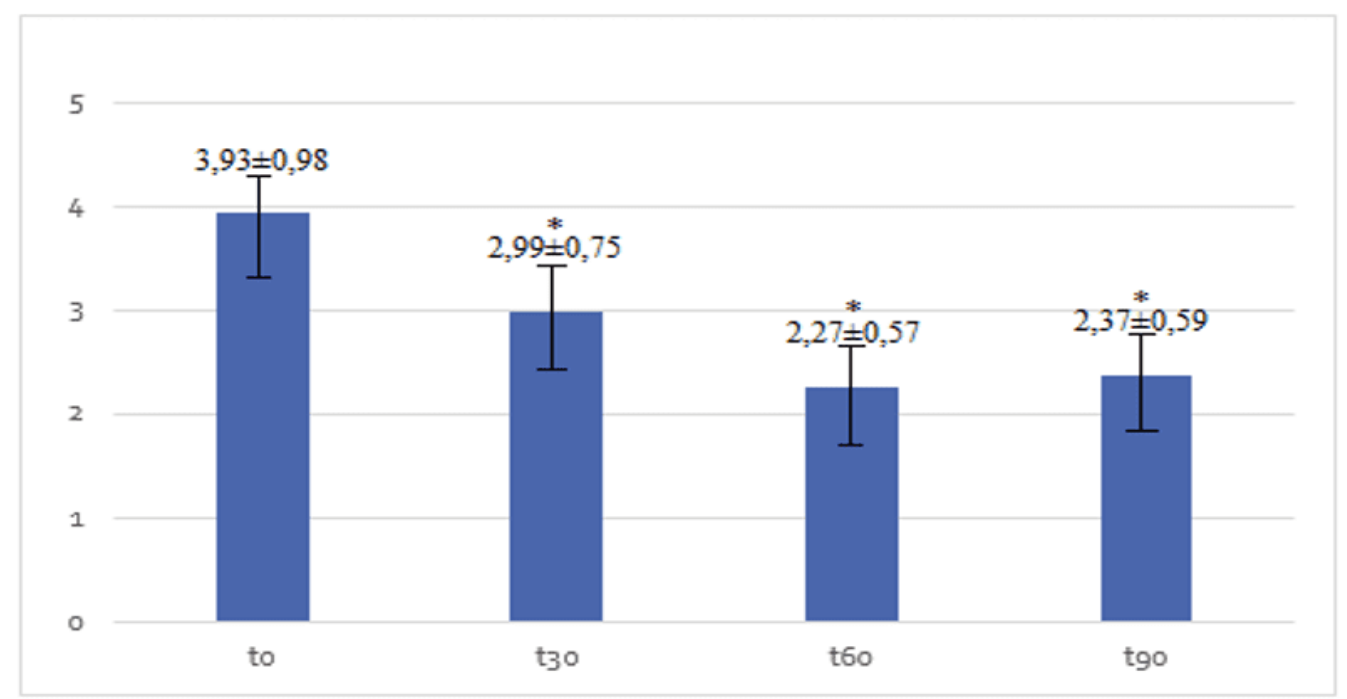

Fonte: dados primários (2018). Legenda: ${ }^{*} \mathrm{p}<0,05$ quando comparado ao t0. 
Figura 3b - Comparação da dor/desconforto no joelho mensurada por Lequesne (Média \pm DP) durante os tempos do estudo com a suplementação do mix de colágeno.

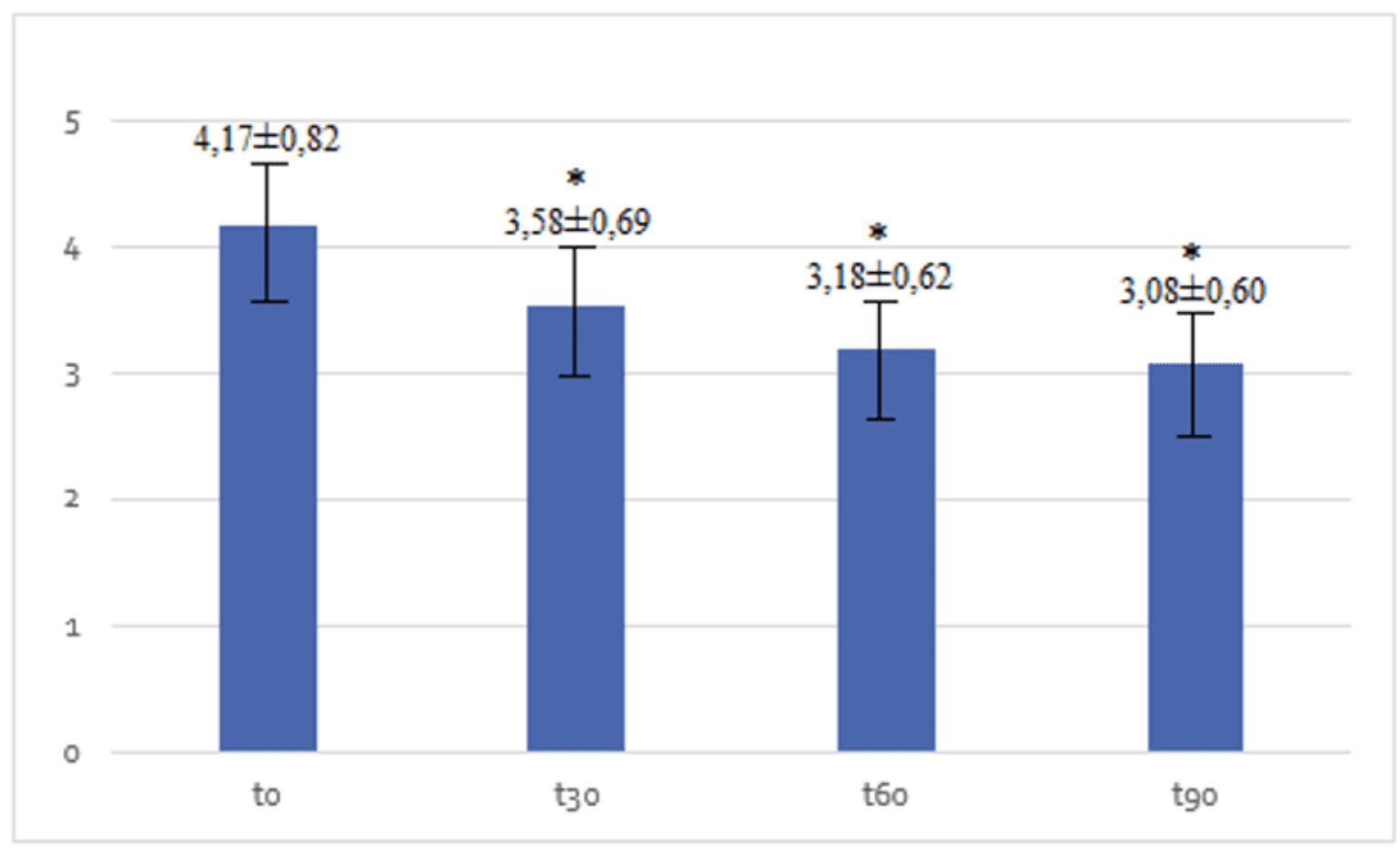

Fonte: dados primários (2018). Legenda: ${ }^{*} \mathrm{p}<0,05$ quando comparado ao t0.

\section{CONSIDERAÇÕES FINAIS}

Os protocolos utilizados neste estudo se mostraram eficazes com resultados como: a melhora da força muscular, diminuição da dor e melhora das atividades diárias de pacientes com dor ou algum desconforto nas articulações. Observou-se que o mix de colágeno pode ser uma boa alternativa para problemas articulares para idosos ativos fisicamente.

Com base nos resultados obtidos conclui-se que o suplemento mix de colágeno composto por peptídeos bioativos de colágeno hidrolisado, colágeno tipo II intacto, silício e nutrientes envolvidos no metabolismo ósseo articular (Duo flex® - marca Snella) demonstrou eficácia nas manifestações articulares evidenciando ser um bom nutracêutico para os idosos ativos. 


\section{REFERÊNCIAS}

ADDOR, F. A. S. A. Influência de um suplemento nutricional com peptídeos de colágeno nas propriedades da derme. Surgical \& Cosmetic Dermatology, v. 7, n. 2, p. 116-122, 2015.

BAKILAN, F. et al. Effects of native type II collagen treatment on knee osteoarthritis: a randomized controlled trial. The Eurasian journal of medicine, v. 48, n. 2, p. 95, 2016.

BARBOSA-SILVA, T. G. et al. Enhancing SARC-F: Improving sarcopenia screening in the clinical practice. Journal of the American Medical Directors Association, v. 17, n. 12, p. 1136-1141, 2016.

BRASIL. Ministério da Saúde. Secretaria de Atenção à Saúde Departamento de Ações Programáticas Estratégicas. Caderneta de Saúde da Pessoa Idosa. 4ª edição. Brasília - DF. 2017.2 Disponível em: (http://bvsms.saude.gov.br/bvs/publicacoes/caderneta_saude_pessoa_idosa_3ed.pd f). Acesso em: 09/06/2018.

CAI, J.; STOYANOV, A. Population aging and comparative advantage. Journal of International Economics, v. 102, p. 1-21, 2016.

CHUMLEA, W. C.; BAUMGARTNER, R. N. Status of anthropometry and body composition data in elderly subjects. The American Journal of Clinical Nutrition, v. 50, n. 5, p. 1158-1166, 1989.

CROWLEY, D. C. et al. Safety and efficacy of undenatured type II collagen in the treatment of osteoarthritis of the knee: a clinical trial. International journal of medical sciences, v. 6, n. 6, p. 312, 2009.

FERNANDES, M. I. Tradução e validação do questionário de qualidade de vida específico para osteoartrose WOMAC (Western Ontario McMaster Universities) para a língua portuguesa. 2002. 119 f. Dissertação (Mestrado em medicina) - Escola 
Paulista de Medicina, São Paulo, 2003. Disponível em: (http://repositorio.unifesp.br/handle/11600/19401). Acesso em: 15/02/2018

GERMANO, M. D. C. M. et al. Colágeno e os benefícios para pele. 2016. Disponível em:

(http://publicacoesacademicas.fcrs.edu.br/index.php/mostracientificafarmacia/article/ view/1226/994). Acesso em: 16/02/2018.

JIANG, J. X. et al. Collagen peptides improve knee osteoarthritis in elderly women: A 6-month randomized, double-blind, placebo-controlled study. Agro Food Industry Hi Tech, v. 25, p. 19-23, 2014. Disponível em: (http://collagen2joint.com.br/site/artigos/6.pdf). Acesso em: 24/02/2018.

LIPSCHITZ, D. A. Screening for nutritional status in the elderly. Primary care, v. 21, n. 1, p. 55-67, 1994.LUGO, J. P.; SAIYED, Z. M.; LANE, N. E. Efficacy and tolerability of an undenatured type II collagen supplement in modulating knee osteoarthritis symptoms: a multicenter randomized, double-blind, placebo-controlled study. Nutrition journal, v. 15, n. 1, p. 14, 2015.

MALMSTROM, T. K. et al. SARC-F: a symptom score to predict persons with sarcopenia at risk for poor functional outcomes. Journal of cachexia, sarcopenia and muscle, v. 7, n. 1, p. 28-36, 2016.

MARX, F. C. et al. Tradução e validação cultural do questionário algofuncional de Lequesne para osteoartrite de joelhos e quadris para a língua portuguesa. Revista Brasileira de Reumatologia, 2006.

MELLO, F. M. Análise da correlação dos escores de atividade de doença na artrite reumatóide. Trabalho de Conclusão do curso de graduação em medicina Universidade Federal de Santa Catarina, Florianópolis, 2008. 66 f. Disponível em: <https://repositorio.ufsc.br/bitstream/handle/123456789/119441/255546.pdf?sequenc e=1). Acesso em: 01/03/2018. 
MORAN, C. J. et al. Restoration of articular cartilage. The Journal of Bone and Joint Surgery, v. 96, n. 4, p. 336-344, 2014.

MOTA, J. P. Características sociodemográficas, fragilidade e sarcopenia em idosos longevos. 2017. Disponível em: (https://bdtd.ucb.br:8443/jspui/handle/tede/2184). Acesso em: 23/02/2018.

OFTADEH, R. et al. Biomechanics and mechanobiology of trabecular bone: a review. Journal of biomechanical engineering, v. 137, n. 1, p. 010802, 2015.

ORGANIZACIÓN PANAMERICANA DE LA SALUD. División de Promoción y Protección de la Salud (HPP). Encuesta Multicentrica salud beinestar y envejecimiento (SABE) em América Latina el Caribe: Informe Preliminar [Internet]. In: XXXVI Reunión del Comité asesor de investigaciones em Salud; 9-11 jun 2001; Kingston, Jamaica: OPAS, 2002 [acesso em 20 out 2018]. Disponível em: (www.opas.org/program/sabe.htm). Acesso em: 21/06/2018.

QUEIROZ, Z. P. V.; LEMOS, N. F. D.; RAMOS, L. R. Fatores potencialmente associados à negligência doméstica entre idosos atendidos em programa de assistência domiciliar. Ciência \& Saúde Coletiva, v. 15, p. 2815-2824, 2010.

SANTOS, A. J. A. O.; MELO, M. W. L.; SOUZA, M. F. C. Avaliação do consumo de alimentos com compostos bioativos e com agentes cancerígenos em pacientes oncológicos. HU Revista, v. 39, n. 3 e 4, 2013. Disponível em: (https://hurevista.ufjf.emnuvens.com.br/ hurevista/article/view/2236/770). Acesso em: 11/05/2018.

UNITED NATIONS. Department of Economic and Social Affairs, World Population Ageing. No. E.17.XIII.3 New York, 2017. Disponível em: (http://www.un.org/en/development/desa/population/publications/pdf/ageing/WPA201 7_Highlights.pdf). Acesso em: 04/07/2018.

VAN VIJVEN, J. P. J. et al. Symptomatic and chondroprotective treatment with collagen derivatives in osteoarthritis: a systematic review. Osteoarthritis and 
cartilage, v. 20, n. 8, p. 809-821, 2012.YAAR, M.; ELLER, M. S.; GILCHREST, B. A. Fifty years of skin aging. In: Journal of Investigative Dermatology Symposium Proceedings. Elsevier, 2002. p. 51-58.ZDZIEBLIK, D. et al. Collagen peptide supplementation in combination with resistance training improves body composition and increases muscle strength in elderly sarcopenic men: a randomised controlled trial. British Journal of Nutrition, v. 114, n. 8, p. 1237-1245, 2015.

ZIEGLER, F. L. F.; SGARBIERI, V. C. Caracterização químico-nutricional de um isolado proteico de soro de leite, um hidrolisado de colágeno bovino e misturas dos dois produtos. Revista de Nutrição, 2009.

Enviado: Março, 2020.

Aprovado: Agosto, 2020. 